\title{
The role of maintenance therapy in eosinophilic esophagitis: who, why, and how?
}

\author{
Hamish Philpott ${ }^{1} \cdot$ Evan S. Dellon $^{2}$ (I)
}

Received: 20 September 2017/ Accepted: 26 September 2017/Published online: 10 October 2017

(C) Japanese Society of Gastroenterology 2017

\begin{abstract}
In patients with eosinophilic esophagitis (EoE) who do not respond to proton pump inhibitors, initial antiinflammatory/anti-eosinophilic treatment is with either topical corticosteroids or dietary elimination. A large body of literature supports the efficacy of these approaches, with histologic response rates in the 50-90\% range for steroids and $70 \%$ range for the six-food elimination diet. However, these studies are almost all short-term and data evaluating long-term safety and efficacy of either treatment are limited. Nevertheless, because EoE is chronic, symptomatic, endoscopic, and histologic disease activity recurs when successful treatments are stopped. An emerging body of data also suggest that left untreated, persistent eosinophilic esophageal inflammation may progress to fibrostenosis over time. Therefore, maintenance therapy in EoE is intuitively attractive. This paper reviews the rationale for maintenance treatment in EoE, the available long-term pharmacologic and dietary response data for EoE, and discusses who may benefit the most from ongoing treatment. While all patients with EoE can be offered maintenance treatment, this option should be strongly recommended in patients with severe disease phenotypes or complications, including malnutrition or failure to thrive, esophageal fibrostenosis, strictures requiring
\end{abstract}

Evan S. Dellon

edellon@med.unc.edu

1 Northern Adelaide Local Health Network (NALHN), Department of Gastroenterology Lyell McEwin and Modbury Hospitals, University of Adelaide, Adelaide, SA, Australia

2 Center for Esophageal Diseases and Swallowing, Division of Gastroenterology and Hepatology, University of North Carolina at Chapel Hill School of Medicine, CB\#7080, Bioinformatics Building, 130 Mason Farm Rd., Chapel Hill, NC 27599-7080, USA dilation, recurrent food bolus impaction, history of perforation, and symptoms that recur quickly after treatment discontinuation. In all EoE patients, regular follow-up is also advised.

Keywords Eosinophilic esophagitis - Treatment . Maintenance $\cdot$ Outcomes $\cdot$ Steroid

\section{Introduction}

Eosinophilic esophagitis (EoE) is a chronic inflammatory condition thought to be caused by exposure to food antigens [1]. Characteristic clinical symptoms in adults include dysphagia and food bolus impaction, due to pathological narrowing and impaired distensibility of the inflamed and fibrosed esophagus [2]. In children, symptoms are thought to reflect eosinophilic inflammatory activity, can be nonspecific, and include heartburn, vomiting/regurgitation, abdominal pain, feeding difficulty, and failure to thrive [3]. Upper gastrointestinal endoscopy and esophageal biopsy demonstrating $>15$ eosinophils per high-power field (HPF) is diagnostic of EoE in a patient with consistent symptoms and the absence of competing disease processes [4].

Treatment of a patient with suspected EoE is initiated with an 8-week trial of twice daily proton pump inhibitor (PPI), with endoscopy and esophageal biopsy then being performed. Those patients who initially had esophageal eosinophilia but have clinical and histological resolution following the PPI are classified with PPI-responsive esophageal eosinophilia (PPI-REE) and thus maintenance treatment with daily PPI is generally advised [4]. Whether PPI-REE is a subtype of GERD, EoE or a combination of 
both is debatable and an area of current controversy (see below), particularly given a response to PPI does not preclude resolution of esophageal eosinophilia with diet or corticosteroids [5-8]. At present, those patients with esophageal eosinophilia ( $>15 / \mathrm{HPF}$ ) that persists after a PPI trial are considered to have confirmed EoE, and the initial treatment options are either topical corticosteroids (tCS) or dietary elimination diet; endoscopic dilatation can be performed in conjunction with these treatments when esophageal narrowing or strictures are noted.

There are currently no approved pharmacologic agents for treatment of EoE, so tCS are medications originally manufactured to treat asthma which are used off-label. Instead of inhaling these, patients can swallow dry powder fluticasone (from a multi-dose inhaler) or mix liquid budesonide (from the nebulized formulation) with sucralose (e.g. 'Splenda') or other thickener to form a slurry [9-13]. Numerous studies in both adult and pediatric cohorts confirm that these medications are effective for achieving histological response or remission in $50-90 \%$ of patients [14-19]. It is important to note, however, that these studies are, for the most part, short-term (2-12 weeks in duration), and more recent ones have tested potential commercial products that have been specifically formulated to treat EoE, but that are not yet approved or available for clinical use $[14,20]$. Safety data are reassuring, the only side effect of tCS of any frequency being esophageal candidiasis $(5-10 \%)$ which is typically asymptomatic and detected incidentally on follow-up endoscopy; oral candidiasis is even less common [21, 22].

Dietary elimination is an effective non-pharmacologic initial treatment for EoE. The most commonly used approach requires strict empirical avoidance of 4-6 common food groups initially (e.g. wheat/gluten, dairy, eggs, soy/legumes, nuts, and seafood), followed by endoscopy and biopsy after 6 weeks [23, 24]. Histological response occurs in approximately $60-70 \%$ of patients, however the absence of reliable allergy tests to guide treatment means that sequential reintroduction of each food every 6 weeks, followed by repeated endoscopy is needed to isolate the culprit food group or groups [10, 25-27]. The absence of allergy tests that also accurately predict food triggers is associated with lower efficacy rates of targeted elimination diets in the 40\% range [23]. In addition to tCS and dietary treatment, esophageal dilation can reliably improve symptoms in $95 \%$ percent of EoE patients with fibrostenotic changes in the esophagus, with repeat dilatation often required within 12 months, and a low risk of perforation $(<0.5 \%)$ [28]. Dilatation does not address the underlying inflammatory process and hence repeated procedures are inevitable if this modality is used alone. However, it is an important therapy to use if esophageal strictures are present.
Whilst effective short-term treatment strategies for EoE exist, the chronic nature of the illness means that treatment cessation almost universally results in disease recurrence $[17,29,30]$. As discussed below, data pertaining to the time course and characteristics of disease recurrence, predictors of disease recurrence, and the potential detrimental effects of treatment cessation in precipitating or perpetuating a severe phenotype or complications of EoE are limited. Finally, balancing the costs and benefits of longterm treatment and defining treatment goals, whether to control symptoms, normalize endoscopic appearance, maintain histological remission (according to esophageal biopsy and eosinophil count), maximize quality of life, improve biomarkers, avoid complications, or all of these, remain key considerations. This paper will review the rationale for maintenance treatment in EoE, discuss which patients with EoE should be considered for long-term treatment, and provide an overview of the data for specific maintenance treatment options.

\section{Rationale for maintenance treatment in $\mathbf{E o E}$}

There are several rationales for maintenance treatment in EoE, including that EoE is a chronic inflammatory condition, and spontaneous resolution is thought to be uncommon, that disease activity recurs when treatments are stopped, and that untreated disease can lead to fibrostenotic complications [31]. Data supporting the chronicity of EoE can be obtained from multiple sources. The best natural history data are derived from Straumann et al. in a prospective case series of 30 adult patients followed for a mean 7.2 years in the absence of anti-inflammatory treatment [32]. They found that symptoms of dysphagia persisted in 29 out 30 subjects, and esophageal eosinophilia persisted in all. All subsequent studies of natural history are either retrospective or make inferences after cessation of treatment. Liacouros et al., in a retrospective study of 381 children with EoE over 10 years, identified a subset of patients treated with a short-term (4 week) course of oral methylprednisolone $(n=39)$ and swallowed fluticasone $(n=17)$, respectively [33]. Esophageal biopsies were performed prior to treatment, after 4 weeks of treatment and again 6 months following treatment cessation. The mean esophageal eosinophil count initially nearly normalized with treatment, but then returned to baseline at 6 months in both groups. Similar data were observed in adults where after successful treatment with tCS, the median time to symptom recurrence was 9 months. Even after a long-term so-called "deep remission" was achieved for more than 2 years in a prospective cohort study, Greuter et al. noted that only 6 of 351 patients (1.7\%) were able to stop tCS without relapse [34-36]. Straumann et al. 
conducted a randomized placebo-controlled study of 50 weeks of maintenance with swallowed budesonide compared with a placebo in a set of patients who had initially had histologic response to budesonide [29]. They demonstrated persistently elevated esophageal eosinophil counts in the placebo compared to the active treatment arm. In addition, in all of the placebo-controlled trials of tCS, levels of esophageal eosinophilia are essentially unchanged in the placebo arms [14, 19, 20, 37]. Results of a study of symptoms as the primary endpoint again showed improvement with corticosteroids followed by relapse of reported dysphagia at 3 years [38]. These data clearly show that while topical steroids are initially effective, if and when they are stopped, EoE disease activity will recur.

As noted above, studies of dietary elimination consistently report endoscopic and clinical remission of approximately $60-70 \%$ in patients on the 6-food elimination diet $[19,25,26]$. When treatment is continued, it is likely that the disease will remain quiescent, although prospective studies spanning multiple years or decades are yet to be reported, but it is clear that when dietary therapy is stopped or food triggers are reintroduced, EoE disease activity also recurs $[25,39]$. A study by Lucendo et al. of the six-food elimination diet provides the most credible long-term remission data in adult patients $[26,29,38]$. They followed initial responders for up to 6 months and those who remained adherent to their diets remained in remission [26]. Additionally, a recent study of more than 1800 children with EoE followed at Children's Hospital of Philadelphia found that only $9(0.5 \%)$ were able to maintain remission long-term without remaining on dietary treatment [40].

In addition to disease recurrence after treatment is stopped, data are emerging that there are complications of EoE related to ongoing eosinophilic inflammation. These complications include strictures, food bolus impaction, esophageal perforation, and (in children especially) poor nutrition and failure to thrive $[17,30,32,41]$. The clinicopathological sequelae of persistent esophageal inflammation include progressive thickening of the lamina propria due to collagen deposition, muscular hyperplasia, and stricture formation, with resultant esophageal narrowing [41]. There are three studies that have assessed this risk in adults. A Swiss study of 200 patients demonstrated that diagnostic delay was the only variable associated with stricture formation [17]. A retrospective study of 379 patients in North Carolina found that older age at diagnosis correlated with a fibrostenotic phenotype, suggesting that EoE is a chronic condition that, untreated, will progress to stricture formation [31]. Finally, Lipka et al. in a retrospective study of 64 adult patients determined that a delay in diagnosis and treatment lead to a progressive stenosing phenotype of EoE, with increased esophageal luminal diameter being inversely associated with treatment delay [30].

The corollary of the observation that treatment delay can lead to progressive esophageal narrowing and stricture is that treatment will prevent these complications. Kuchen et al., using prospectively acquired data from 206 adults with EoE (33 of whom developed a food bolus impaction after a mean follow-up of 5 years), showed the treatment with topical corticosteroids conferred a reduced risk of subsequent food bolus impaction (OR 0.41), whilst oesophageal stricture was associated with increased risk (OR 2.66) [17]. Nicodeme et al., in a prospective study of 70 adult patients that utilized endoscopic high-resolution impedance planimetry to measure esophageal distensibility (EndoFLIP), found that distensibility was impaired at baseline in the 12 patients that went on to develop food bolus impaction [42]. Similarly, patients requiring dilatation for stricture had impaired distensability [42]. In a follow-up study from the same group, Carson et al. showed that improvements in esophageal distensibility, approximately equivalent to a 2-mm increase in luminal diameter, could by achieved with either tCS or dietary therapy in the absence of esophageal dilation [43]. Taken in the context of their prior data, it can be extrapolated that this improvement could explain the rates of decreased food impactions. Finally, in a study of EoE patients who required dilation at baseline for esophageal stricture or narrowing, Runge et al. found that those who had initially histologic response needed less than half as many dilations over a mean follow-up time of 19 months compared to histologic non-responders [31, 44]. Thus, in addition to decreased risk for food impaction, successful treatment decreases the need for dilations in patients who already have fibrostenotic complications.

\section{Which patients should be considered for maintenance treatment?}

Because of the limited amount of long-term data on EoE, recommendations vary for the role of maintenance therapy. The American College of Gastroenterology clinical guideline for the management of patients with EoE (published in 2013) states that maintenance treatment using tCS and/or diet can be considered for all patients ('strong recommendation, moderate evidence'), especially those with stricture, food impaction, or recurrent symptoms off treatment ('conditional recommendation, low evidence') [4]. The EoE working group of the European Society for Paediatric Gastroenterology Hepatology and Nutrition (ESPHAGN) issued a medical position paper (2014) detailing the few studies relating to maintenance tCS, and discussed a regimen where, following the resolution of 
symptoms and with confirmed histological remission, the dose is titrated downwards and ceased [45]. Symptoms alone are suggested as a reason to repeat endoscopy and to reintroduce therapy. Treatment of asymptomatic patients is suggested to be individualized and according to local practice. No specific statement is made regarding dietary therapy (although this is presumably a long-term strategy). Finally, guidelines were published in 2017 by a European working group [comprised of members of ESPHAGN, the European Academy of Allergy and Clinical Immunology (EAACI), the European Society of Eosinophilic Esophagitis (EUREOS), and United European Gastroenterology (UEG)] that suggest maintenance treatment using an 'effective anti-inflammatory drug or diet' be considered for all patients [7]. This guideline, similar to the two earlier papers, concludes that evidence supporting maintenance treatment is limited, but that expert opinion primarily supports this strategy [7]. Indeed, $100 \%$ of experts on the panel agreed that maintenance corticosteroids were effective and safe, but a specific analysis of the efficacy and safety of dietary measures as maintenance was not detailed.

A reasonable synthesis of the clinical guidelines to date is that evidence supporting maintenance therapy is limited, but that experts advocate treatment for most or all patients with EoE. The belief is that if EoE is untreated, many patients will suffer progressive disease with luminal narrowing, stricture, and food bolus impaction (or associated and most ominously of all, esophageal perforation) [31]. The end-point of treatment is therefore currently clinical (to prevent symptoms of dysphagia, to prevent food bolus impaction, and to allow adequate nutritional intake) and perhaps endoscopic (to prevent stricture formation). In this context, patients who have fibrostenosis, have previously needed esophageal dilatation, have suffered food bolus impaction or perforation, have had malnutrition, or have rapidly recurring symptoms after treatment is stopped should be most strongly encouraged to maintain treatment. However, it is important to acknowledge in EoE that symptoms are an imprecise guide to disease activity [46] and that some patients can have an 'all or nothing' phenomenon presenting only as recurrent food bolus impaction. Therefore, most experts feel that a goal of maintenance therapy should also be to improve or normalize histology, which requires surveillance endoscopy given the lack of non-invasive measure of disease activity. However, at present time we do not have substantial data linking histologic response to important clinical outcomes such as decreased complications.

\section{Maintenance therapy and long-term outcomes}

The current lack of data relating to long-term maintenance efficacy, the ability of pharmacotherapies to cause side effects, and the possibility of poor compliance or malnutrition with dietary therapy, all sensibly point to a need for a structured surveillance program. Use of the lowest effective dose of anti-inflammatory medication, and routine clinical review possibly with the use of a structured symptom score at pre-set intervals, and consideration of interval endoscopy to determine histological remission in patients with a high-risk history (e.g. those with recurrent food bolus impaction, esophageal perforation, or failure to thrive) may be beneficial, although remain untested. Clinical guidelines are therefore not proscriptive in terms of the ideal follow-up for patients with EoE on maintenance treatment. Ultimately, given the rapid evolution of diagnostic techniques (for example, unsedated transnasal endoscopy, the esophageal string test, and the cytosponge) and pharmacotherapies, regular review may facilitate timely access to the best treatment [14, 47-50]. In patients that do decide to cease treatment, even greater vigilance to detect symptomatic or histological recurrence seems prudent.

Maintaining the lowest effective dose of medication possible in EoE makes intuitive sense given the potential long-term side effects of corticosteroids (e.g. adrenal suppression, oropharyngeal candidiasis) [51] and the unknown safety profile of high-dose proton pump inhibitors. Proton pump inhibitors may be reduced from twice daily 'highdose' to daily dosing at approved dosing levels with maintenance of histological remission in $75-85 \%$ of patients at up to 1 year of follow-up [52, 53]. Butz et al. found that tCS (fluticasone) may be reduced from 1760 mcg daily to 880 mcg daily with remission defined as $<1$ eosinophil per high-power field maintained in 73\% [13]. Miehlke et al. found budesonide $2 \mathrm{mg}$ daily was equally efficacious to $4 \mathrm{mg}$ daily [20]. Straumann et al. performed a randomized trial of the longest tCS treatment to date (12 months), but a daily dose of $0.5 \mathrm{mg}$ of budesonide was not adequate to maintain long-term histologic remission in most patients [29]. The same group evaluated long-term tCS treatment as well, and found that these medications tended to be safe and effective [34]. There have been two long-term follow-up studies of children treated with maintenance topical steroids, one with fluticasone and one with budesonide, and these were generally effective for maintaining responses $[38,41]$. However, there are some emerging data that tCS may lose some efficacy from the histologic response standpoint over time. Eluri et al. performed a retrospective cohort study of 55 patients treated with tCS (budesonide or fluticasone) followed up for a 
mean of 11.4 months and with an average of 2 follow-up endoscopies [15]. Surprisingly, $61 \%$ of patients had histological loss of response, though patients who maintained their initial dose were less likely to relapse than those in whom the dose was reduce (OR 0.10) [15]. In an openlabel extension of a randomized controlled trial of budesonide oral suspense, $42 \%$ of patients maintained histologic response on a 2-mg daily dose, after previously achieving response on a 2-mg twice daily dose [54]. While acknowledging current knowledge gaps, a reasonable synopsis of the current evidence is that budesonide $2 \mathrm{mg}$ daily appears adequate and should be maintained, and that given a single trial only has suggested some maintenance of efficacy with dose reduction of fluticasone, that dose reduction below $1760 \mathrm{mcg}$ could be considered with close monitoring [13]. For all long-term medication approaches, careful follow-up is essential.

Because it avoids the potential side-effects and dosing issues of long-term pharmacologic use, dietary therapy is an appealing maintenance treatment strategy. Once remission is achieved and serial food re-introduction with endoscopy has established the 'permanent' diet (which will most commonly involve avoidance of 1-3 food groups, most often wheat/ gluten and dairy), the ever present challenge remains compliance [10,55-57]. Data regarding the long-term efficacy of dietary avoidance is minimal, and, as a result, it cannot be stated for certainty that even with the strictest adherence, that food avoidance will result in prolonged remission. Nonetheless, Philpott et al. found that of the 18 patients who had initially sustained a remission after 3 months of dietary therapy, that at 9 months, $7 / 18$ (or 39\%) had ceased treatment [10]. More encouraging was that of the 10/18 (55\%) who continued the diet, all 10 were in remission at 9 months (as defined by endoscopy and biopsy [10]. Lucendo et al. found that of 42 patients who initially sustained a remission with dietary avoidance, 25 were in remission at 12 months (many dropped out of the study), 15 were in remission at 2 years, and 4 were in remission at 3 years [26]. Similarly, a recent study by Reed et al. has shown that of 21 patients who were initially in remission and had food trigger identification, the 10 who remained adherent to the diet maintained remission at a mean of 25 months of follow-up; the other 11 subjects had recurrent disease activity with food reintroduction or were non-adherent [58]. Failure to return for follow-up and dietary non-compliance, rather than a lack of continued response to diet, likely explains the decrement in the number of patients in remission [26]. Thus, it appears based on current data that dietary therapy does result in durable disease control, but that compliance may be difficult thereby limiting the long-term efficacy of this treatment modality.

Finally, esophageal dilation can be used to treat stricture and effectively alleviate dysphagia. Most often, it is advocated for use in combination with adjunctive anti-inflammatory tCS or dietary therapy, or when one or both have failed [7]. Initial safety concerns have largely been alleviated, dilatation being considered safe and generally well-tolerated, the major side effect being peri- and postprocedural, and oesophageal perforation being very rare $(<0.4 \%)$ [28]. Most patients will require repeated dilation ( 2 or more), and of these patients most will have the repeat procedure within 12 months [59]. If used as a monotherapy, repeated procedures are likely required as the underlying inflammatory process is not addressed [7, 60, 61].

\section{Conclusions}

EoE is a chronic inflammatory condition and symptoms, endoscopic features, and histologic findings all recur if initial successful treatment is stopped. A growing body of evidence suggests that esophageal fibrostenosis may develop in many patients with EoE if it is not treated, and that treatment with tCS decreases the risk of esophageal stricture, food bolus impaction, and need for dilation. The ability of tCS and dietary therapy to induce short-term histological remission has led to clinical guidelines advocating maintenance therapy with either treatment. While this makes intuitive sense, the recommendation is largely based on expert option as there is a lack of high-quality long-term prospective studies. Not all patients will wish to maintain treatment, nor will all be complaint with their doctor's advice. A common-sense position given these practical obstacles may be to most strongly advocate maintenance therapy in those with established fibrostenosis, strictures that have required dilation, recurrent food bolus impaction, history of esophageal perforation, or malnutrition/failure to thrive (most commonly seen in children). In the pediatric population, the hope would be that long-term successful maintenance treatment could lead to decreased EoE complications and more mild disease activity in the future. Key unanswered questions include how to predict which patients are likely to progress to structuring disease without treatment, if response to tCS or dietary treatment will be durable (in the compliant patient), if complete histological remission is necessary to prevent disease progression, and whether routine surveillance endoscopy is necessary. Future technological innovation allowing minimally invasive sampling of esophageal mucosa may provide key insights into disease pathogenesis and facilitate tailored maintenance treatment strategies.

\section{Compliance with ethical standards}

Funding sources Dr. Hamish Philpott received finding from AusEE, and from the Philip Bushell Foundation to support this project. Dr. Dellon received funding from NIH award R01 DK101856 as well as 
U54AI117804 (CEGIR), which is part of the Rare Disease Clinical Research Network (RDCRN), an initiative of the Office of Rare Disease Research (ORDR), NCATS, and is funded through collaboration between NIAID, NIDDK, and NCATS.

Disclosures Dr. Dellon is a consultant for Adare, Alivio, Banner, Enumeral, GSK, Cellgene/Receptos, Regeneron, and Shire. Dr. Dellon has received research funding from Adare, Meritage, Miraca Life Sciences, Nutricia, Celegene/Receptos, Regeneron, and Shire, and an educational grant from Banner. Dr Philpott has received educational assistance from Shire and Aspen.

Conflict of interest The authors declare that they have no conflict of interest.

\section{References}

1. Rothenberg ME. Molecular, genetic, and cellular bases for treating eosinophilic esophagitis. Gastroenterology. 2015;148(6):1143-57. doi:10.1053/j.gastro.2015.02.002.

2. Philpott H, Nandurkar S, Thien F, et al. Eosinophilic esophagitis: a clinicopathological review. Pharmacol Ther. 2015;146:12-22. doi:10.1016/j.pharmthera.2014.09.001.

3. Dellon ES, Liacouras CA. Advances in clinical management of eosinophilic esophagitis. Gastroenterology. 2014;147(6):1238-54. doi:10.1053/j.gastro.2014.07.055.

4. Dellon ES, Gonsalves N, Hirano I, Liacouras CA, Katzka DA, et al. ACG clinical guideline: evidenced based approach to the diagnosis and management of esophageal eosinophilia and eosinophilic esophagitis (EoE). Am J Gastroenterol. 2013;108(5):679-92. doi:10.1038/ajg.2013.71 (quiz 93).

5. Lucendo AJ, Arias A, Gonzalez-Cervera J, et al. Dual response to dietary/topical steroid and proton pump inhibitor therapy in adult patients with eosinophilic esophagitis. J Allergy Clin Immunol. 2016;137(3):931-4 e2. doi:10.1016/j.jaci.2015.07.033.

6. Molina-Infante J, Bredenoord AJ, Cheng E, et al. Proton pump inhibitor-responsive oesophageal eosinophilia: an entity challenging current diagnostic criteria for eosinophilic oesophagitis. Gut. 2016;65(3):524-31. doi:10.1136/gutjnl-2015-310991.

7. Lucendo AJ, Molina-Infante J, Arias A, et al. Guidelines on eosinophilic esophagitis: evidence-based statements and recommendations for diagnosis and management in children and adults. United Eur Gastroenterol J. 2017;5(3):335-58. doi:10.1177/ 2050640616689525.

8. Sodikoff J, Hirano I. Proton pump inhibitor-responsive esophageal eosinophilia does not preclude food-responsive eosinophilic esophagitis. J Allergy Clin Immunol. 2016;137(2):631-3. doi:10. 1016/j.jaci.2015.07.008.

9. Dellon ES, Sheikh A, Speck O, et al. Viscous topical is more effective than nebulized steroid therapy for patients with eosinophilic esophagitis. Gastroenterology. 2012;143(2):321-4 e1. doi:10.1053/j.gastro.2012.04.049.

10. Philpott H, Nandurkar S, Royce SG, et al. A prospective open clinical trial of a proton pump inhibitor, elimination diet and/or budesonide for eosinophilic oesophagitis. Aliment Pharmacol Ther. 2016;43(9):985-93. doi:10.1111/apt.13576.

11. Aceves SS, Bastian JF, Newbury RO, et al. Oral viscous budesonide: a potential new therapy for eosinophilic esophagitis in children. Am J Gastroenterol. 2007;102(10):2271-9. doi:10.1111/ j.1572-0241.2007.01379.x (quiz 80).

12. Konikoff MR, Noel RJ, Blanchard C, et al. A randomized, double-blind, placebo-controlled trial of fluticasone propionate for pediatric eosinophilic esophagitis. Gastroenterology. 2006;131(5):1381-91. doi:10.1053/j.gastro.2006.08.033.
13. Butz BK, Wen T, Gleich GJ, et al. Efficacy, dose reduction, and resistance to high-dose fluticasone in patients with eosinophilic esophagitis. Gastroenterology. 2014;147(2):324-33 e5. doi:10. 1053/j.gastro.2014.04.019.

14. Dellon ES, Katzka DA, Collins MH, et al. Budesonide oral suspension improves symptomatic, endoscopic, and histologic parameters compared with placebo in patients with eosinophilic esophagitis. Gastroenterology. 2017;152(4):776-86 e5. doi:10. 1053/j.gastro.2016.11.021.

15. Eluri S, Runge TM, Hansen J, et al. Diminishing effectiveness of long-term maintenance topical steroid therapy in PPI non-responsive eosinophilic esophagitis. Clin Transl Gastroenterol. 2017;8(6):e97. doi:10.1038/ctg.2017.27.

16. Remedios M, Campbell C, Jones DM, et al. Eosinophilic esophagitis in adults: clinical, endoscopic, histologic findings, and response to treatment with fluticasone propionate. Gastrointest Endosc. 2006;63(1):3-12. doi:10.1016/j.gie.2005.07.049.

17. Kuchen T, Straumann A, Safroneeva E, et al. Swallowed topical corticosteroids reduce the risk for long-lasting bolus impactions in eosinophilic esophagitis. Allergy. 2014;69(9):1248-54. doi:10. 1111/all.12455.

18. Murali AR, Gupta A, Attar BM, et al. Topical steroids in eosinophilic esophagitis: systematic review and meta-analysis of placebo-controlled randomized clinical trials. J Gastroenterol Hepatol. 2016;31(6):1111-9. doi:10.1111/jgh.13281.

19. Cotton CC, Eluri S, Wolf WA, et al. Six-food elimination diet and topical steroids are effective for eosinophilic esophagitis: a meta-regression. Dig Dis Sci. 2017;. doi:10.1007/s10620-0174642-7.

20. Miehlke S, Hruz P, Vieth M, et al. A randomised, double-blind trial comparing budesonide formulations and dosages for shortterm treatment of eosinophilic oesophagitis. Gut. 2016;65(3):390-9. doi:10.1136/gutjnl-2014-308815.

21. Sodikoff J, Hirano I. Therapeutic strategies in eosinophilic esophagitis: induction, maintenance and refractory disease. Best Pract Res Clin Gastroenterol. 2015;29(5):829-39. doi:10.1016/j. bpg.2015.09.002.

22. Doerfler B, Bryce P, Hirano I, et al. Practical approach to implementing dietary therapy in adults with eosinophilic esophagitis: the Chicago experience. Dis Esophagus. 2015;28(1):42-58. doi:10.1111/dote.12175.

23. Lucendo AJ. Meta-analysis-based guidance for dietary management in eosinophilic esophagitis. Curr Gastroenterol Rep. 2015;17(10):464. doi:10.1007/s11894-015-0464-y.

24. Cotton CC, Erim D, Eluri S, et al. Cost utility analysis of topical steroids compared with dietary elimination for treatment of eosinophilic esophagitis. Clin Gastroenterol Hepatol. 2017;15(6):841-9 e1. doi:10.1016/j.cgh.2016.11.032.

25. Gonsalves N, Yang GY, Doerfler B, et al. Elimination diet effectively treats eosinophilic esophagitis in adults; food reintroduction identifies causative factors. Gastroenterology. 2012;142(7):1451-9 e1. doi:10.1053/j.gastro.2012.03.001 (quiz e14-5).

26. Lucendo AJ, Arias A, Gonzalez-Cervera J, et al. Empiric 6-food elimination diet induced and maintained prolonged remission in patients with adult eosinophilic esophagitis: a prospective study on the food cause of the disease. J Allergy Clin Immunol. 2013;131(3):797-804. doi:10.1016/j.jaci.2012.12.664.

27. Kagalwalla AF, Wechsler JB, Amsden K, et al. Efficacy of a 4-food elimination diet for children with eosinophilic esophagitis. Clin Gastroenterol Hepatol. 2017;. doi:10.1016/j.cgh.2017.05. 048.

28. Dougherty M, Runge TM, Eluri S, et al. Esophageal dilation with either bougie or balloon technique as a treatment for eosinophilic esophagitis: a systematic review and meta-analysis. Gastrointest Endosc. 2017;. doi:10.1016/j.gie.2017.04.028. 
29. Straumann A, Conus S, Degen L, et al. Long-term budesonide maintenance treatment is partially effective for patients with eosinophilic esophagitis. Clin Gastroenterol Hepatol. 2011;9(5):400-9 e1. doi:10.1016/j.cgh.2011.01.017.

30. Lipka S, Kumar A, Richter JE. Impact of diagnostic delay and other risk factors on eosinophilic esophagitis phenotype and esophageal diameter. J Clin Gastroenterol. 2016;50(2):134-40. doi:10.1097/MCG.0000000000000297.

31. Dellon ES, Hirano I. Epidemiology and natural history of eosinophilic esophagitis. Gastroenterology. 2017;. doi:10.1053/j.gas tro.2017.06.067.

32. Straumann A. The natural history and complications of eosinophilic esophagitis. Gastrointest Endosc Clin N Am. 2008;18(1):99-118. doi:10.1016/j.giec.2007.09.009 (ix).

33. Liacouras CA, Spergel JM, Ruchelli E, et al. Eosinophilic esophagitis: a 10-year experience in 381 children. Clin Gastroenterol Hepatol. 2005;3(12):1198-206.

34. Greuter T, Bussmann C, Safroneeva E, et al. Long-term treatment of eosinophilic esophagitis with swallowed topical corticosteroids: development and evaluation of a therapeutic concept. Am J Gastroenterol. 2017; doi:10.1038/ajg.2017.202.

35. Helou EF, Simonson J, Arora AS. 3-yr-follow-up of topical corticosteroid treatment for eosinophilic esophagitis in adults. Am J Gastroenterol. 2008;103(9):2194-9. doi:10.1111/j.15720241.2008.01989.x.

36. Runge TM, Eluri S, Cotton CC, Woosley JT, Shaheen NJ, et al. Causes and outcomes of esophageal perforation in eosinophilic esophagitis. J Clin Gastroenterol. 2016;. doi:10.1097/MCG. 0000000000000718.

37. Gupta SK, Vitanza JM, Collins MH. Efficacy and safety of oral budesonide suspension in pediatric patients with eosinophilic esophagitis. Clin Gastroenterol Hepatol. 2015;13(1):66-76 e3. doi:10.1016/j.cgh.2014.05.021.

38. Andreae DA, Hanna MG, Magid MS, et al. Swallowed fluticasone propionate is an effective long-term maintenance therapy for children with eosinophilic esophagitis. Am J Gastroenterol. 2016;111(8):1187-97. doi:10.1038/ajg.2016.238.

39. Peterson KA, Byrne KR, Vinson LA, et al. Elemental diet induces histologic response in adult eosinophilic esophagitis. Am J Gastroenterol. 2013;108(5):759-66. doi:10.1038/ajg.2012.468.

40. Ruffner MA, Brown-Whitehorn TF, Verma R, et al. Clinical tolerance in eosinophilic esophagitis. J Allergy Clin Immunol Pract. 2017; . doi:10.1016/j.jaip.2017.06.035.

41. Rajan J, Newbury RO, Anilkumar A, et al. Long-term assessment of esophageal remodeling in patients with pediatric eosinophilic esophagitis treated with topical corticosteroids. J Allergy Clin Immunol. 2016;137(1):147-56 e8. doi:10.1016/j.jaci.2015.05.045.

42. Nicodeme F, Hirano I, Chen J, et al. Esophageal distensibility as a measure of disease severity in patients with eosinophilic esophagitis. Clin Gastroenterol Hepatol. 2013;11(9):1101-7 e1. doi:10.1016/j.cgh.2013.03.020.

43. Carlson DA, Lin Z, Hirano I, et al. Evaluation of esophageal distensibility in eosinophilic esophagitis: an update and comparison of functional lumen imaging probe analytic methods. Neurogastroenterol Motil. 2016;. doi:10.1111/nmo.12888.

44. Runge TM, Eluri S, Woosley JT, Shaheen NJ, Dellon ES. Control of inflammation decreases the need for subsequent esophageal dilation in patients with eosinophilic esophagitis. Dis Esophagus. 2017;30(7):1-7.

45. Papadopoulou A, Koletzko S, Heuschkel R, et al. Management guidelines of eosinophilic esophagitis in childhood. J Pediatr Gastroenterol Nutr. 2014;58(1):107-18. doi:10.1097/MPG. 0b013e3182a80be1.

46. Safroneeva E, Straumann A, Coslovsky M, et al. Symptoms have modest accuracy in detecting endoscopic and histologic remission in adults with eosinophilic esophagitis. Gastroenterology. 2016;150(3):581-90 e4. doi:10.1053/j.gastro.2015.11.004.

47. Philpott H, Nandurkar S, Royce SG, et al. Ultrathin unsedated transnasal gastroscopy in monitoring eosinophilic esophagitis. J Gastroenterol Hepatol. 2016;31(3):590-4. doi:10.1111/jgh. 13173.

48. Katzka DA, Geno DM, Ravi A, et al. Accuracy, safety, and tolerability of tissue collection by cytosponge vs endoscopy for evaluation of eosinophilic esophagitis. Clin Gastroenterol Hepatol. 2015;13(1):77-83 e2. doi:10.1016/j.cgh.2014.06.026.

49. Furuta GT, Kagalwalla AF, Lee JJ, et al. The oesophageal string test: a novel, minimally invasive method measures mucosal inflammation in eosinophilic oesophagitis. Gut. 2013;62(10):1395-405. doi:10.1136/gutjnl-2012-303171.

50. Katzka DA, Smyrk TC, Alexander JA, et al. Accuracy and safety of the cytosponge for assessing histologic activity in eosinophilic esophagitis: a two-center study. Am J Gastroenterol. 2017; doi:10.1038/ajg.2017.244.

51. Harel S, Hursh BE, Chan ES, et al. Adrenal insufficiency exists for both swallowed budesonide and fluticasone propionate in the treatment of eosinophilic esophagitis. J Pediatr. 2016;174:281. doi:10.1016/j.jpeds.2016.02.056.

52. Gomez-Torrijos E, Garcia-Rodriguez R, Castro-Jimenez A, et al. The efficacy of step-down therapy in adult patients with proton pump inhibitor-responsive oesophageal eosinophilia. Aliment Pharmacol Ther. 2016;43(4):534-40. doi:10.1111/apt.13496.

53. Molina-Infante J, Rodriguez-Sanchez J, Martinek J, et al. Longterm loss of response in proton pump inhibitor-responsive esophageal eosinophilia is uncommon and influenced by CYP2C19 genotype and rhinoconjunctivitis. Am J Gastroenterol. 2015;110(11):1567-75. doi:10.1038/ajg.2015.314.

54. Dellon ESKD, Collins MH, Hamdi M, et al. Safety and efficacy of oral budesonide suspension for maintenance therapy in eosinophilic esophagitis: results from a prospective open-label study of adolescents and adults. Gastroenterology. 2016;150(Suppl 1):S188 (Ab 953).

55. Lucendo AJ, Arias A. Treatment of adult eosinophilic esophagitis with diet. Dig Dis. 2014;32(1-2):120-5. doi:10.1159/000357088.

56. Molina-Infante J, Arias A, Barrio J, et al. Four-food group elimination diet for adult eosinophilic esophagitis: a prospective multicenter study. J Allergy Clin Immunol. 2014;134(5):1093-9 e1. doi:10.1016/j.jaci.2014.07.023.

57. Arias A, Gonzalez-Cervera J, Tenias JM, et al. Efficacy of dietary interventions for inducing histologic remission in patients with eosinophilic esophagitis: a systematic review and meta-analysis. Gastroenterology. 2014;146(7):1639-48. doi:10.1053/j.gastro. 2014.02.006.

58. Reed CCFC, Koutlas NT, Shaheen NJ et al. Food elimination diets are effective for long-term treatment of adults with eosinophilic oesophagitis. Aliment Pharmacol Ther. 2017 (in press).

59. Runge TM, Eluri S, Cotton CC, et al. Outcomes of esophageal dilation in eosinophilic esophagitis: safety, efficacy, and persistence of the fibrostenotic phenotype. Am J Gastroenterol. 2016;111(2):206-13. doi:10.1038/ajg.2015.399.

60. Schoepfer AM, Gonsalves N, Bussmann C, et al. Esophageal dilation in eosinophilic esophagitis: effectiveness, safety, and impact on the underlying inflammation. Am J Gastroenterol. 2010;105(5):1062-70. doi:10.1038/ajg.2009.657.

61. Lipka S, Keshishian J, Boyce HW, et al. The natural history of steroid-naive eosinophilic esophagitis in adults treated with endoscopic dilation and proton pump inhibitor therapy over a mean duration of nearly 14 years. Gastrointest Endosc. 2014;80(4):592-8. doi:10.1016/j.gie.2014.02.012. 